\title{
PSYCHOSOCIAL THERAPIES FOR CHRONIC HEADACHE
}

Psychosocial interventions in the management of recurrent headache disorders are reviewed from Ohio University, Athens, $\mathrm{OH}$, and University of Mississippi Medical Center, Jackson, MS. The most frequently used interventions in adult patients are: 1) relaxation training, 2) biofeedback, 3) stress-management (cognitive-behavioral) therapy, and 4) dietary modification. Three types of relaxation training are employed: a) progressive muscle relaxation-alternately tensing and relaxing selected muscle groups, b) autogenic training-use of self-instructions of warmth and heaviness to promote relaxation, and c) meditation - use of a silently repeated word or sound to promote mental relaxation. Cognitive-behavioral interventions teach patients to identify' stressful precipitants and strategies for coping with stress. Behavioral interventions yielded outcomes roughly equivalent to those obtained with propanolol in 60 clinical trials and 2400 patients with migraine. More than $50 \%$ improvement was obtained with either relaxation/biofeedback training or propanolol, compared to $14 \%$ with placebo and $3 \%$ if untreated. (Holroyd KA, Penzien DB. Psychosocial interventions in the management of recurrent headache disorders 1: Overview and effectiveness. Behav Med Summer 1994;20:53-62). (Reprints: Kenneth A Holroyd PhD, Dept of Psychology and Institute of Health and Behavioral Sciences, Ohio University, Athens, OH 45701).

COMIENT. The management of headache in children is different from that of adults and requires an assessment of cognitive and affective development and a knowledge of children's concepts of illness and pain. The use of long-term investigational medications in children is often undesirable and relaxation techniques and dietary modification may be more effective and appropriate. (Ped Neur Briefs April 1991; Progress in Pediatric Neurology II, 1994, p 170).

\section{CONGENITAL AND MENTAL RETARDATION SYNDROMES}

\section{WIEDEMANN SYNDROME}

A boy, aged 2 years 5 months, with microcephaly, large anterior fontanelle, delayed psychomotor development, micropenis, and anomalies of thumbs and halluces is reported from the Department of Medical Genetics, Belfast City Hospital, Northern Ireland. From 9 months of age, he had generalized clonic convulsions, and at 18 months he developed minor seizures. An EEG at 13 months showed prominent delta activity and runs of low voltage fast, but no hypsarryhthmia or other seizure patterns. He was never able to sit and he had no speech. Muscle tone was increased, reflexes were hyperactive, and plantar responses extensor. Eye movements were roving. Fundi were normal. Head circumference was normal (50th centile) at birth and fell to below the 2 nd centile within a year. Chromosome analysis showed a 46, XY karyotype. Renal ultrasound revealed an absent left kidney. CT of head at 22 months showed enlargement of all ventricles and cisterns and hypoplasia of the vermis. At 2 years 9 months he became comatose and he died at 3 years. Autopsy was refused. A maternal aunt had short broad thumbs, but no other congenital or genetic disorders were found in the family. Using the criteria microcephaly, short thumbs, micropenis, and mental retardation, the London Dy'smorphology Database selected only Wiedemann's syndrome in diagnosis. Smith-Lemli-Opitz syndrome was also considered. (Nevin NC et al. Microcephaly with large anterior fontanelle, generalized convulsions, 
micropenis, and distinct anomalies of the hands and feet. Another example of Wiedemann syndrome? Clin Genet Aug 1994;46:205-208). (Respond: Professor NC Nevin, Department of Medical Genetics, Floor A, Lisburn Road, Belfast BT9 7AB, UK).

COMMENT. Wiedemann and associates (1985) described 2 males, first cousins, with similar characteristics, whose mothers and maternal grandfather had short broad thumbs and halluces. The present case, the second report of this syndrome, had normal parents. Autosomal or Xlinked dominant inheritance was thought to be most consistent with the findings.

\section{LIFE EXPECTANCY WITH CEREBRAL PALSY}

The life expectancy of children with idiopathic cerebral palsy born during 1966-84 to mothers resident in Mersey region has been analysed at the Department of Public Health, University of Liverpool, UK. Among 1251 subjects traced, one third had quadriplegia, one third hemiplegia, a quarter had diplegia, and the remainder dyskinesia and ataxia. A quarter had severe ambulatory disability, a fifth severe manual disability, and a third an IQ of 50 or less; $11 \%$ had died. One third had a birth weight of $2500 \mathrm{~g}$ or less, and one third a gestational age of 37 weeks or less. Those with normal birth weight had the highest proportion of severe disabilities. About $85-90 \%$ of subjects with CP survived to 20 years compared to $97 \% 20$ year survival in the general population in 1970-2. Subjects with mild functional disabilities (ambulation, manual dexterity, and IQ deficits) had 20 year survival of $99 \%$, while those severely disabled had a 50\% 20 year survival. Birth weight and gestational age were less predictive of survival than functional disability. (Hutton JL et al. Life expectancy in children with cerebral palsy. BMI 13 August 1994;309:431435). (Respond: Professor Peter OD Pharoah, Department of Public Health, University of Liverpool, Liverpool L69 3BX, UK).

COMMENT. One-half of severely disabled cerebral palsied children survived to age 20 , and the life expectancy to 20 years of mild to moderately disabled children was not much lower than that of unaffected children. As more severely affected low birth weight infants survive with advances in neonatal care, future cohorts may show a higher proportion of severely disabled with $\mathrm{CP}$ and a life expectancy approaching that of the normal birth weight CP children. The social, educational, health service, and medico-legal aspects of these findings are noted. A much shorter life expectancy is reported in US studies.

\section{BRAIN DEVELOPMENT MEASURED BY MRI}

MRI data read as normal on 88 male and female patients aged 3 months to 30 years and on 73 healthy male volunteers aged 21 to 70 years were quantified and the volumes of cortical white matter, gray matter, and CSF were computed in a study at the Department of Veterans Affairs Medical Center, and the Department of Psychiatry and Behavioral Science, Stanford University School of Medicine, CA. In the younger samples, obtained from four California clinics, intracranial volume increased by about $300 \mathrm{ml}$ from 3 months to 10 years. Head size of boys was larger than that of girls by about $70 \mathrm{~mL}$, but both sexes followed the same growth trend. Cortical gray matter volume peaked at age 4 years and decreased thereafter; cortical white matter volume increased steadily until age 20 years: cortical and ventricular CSF volumes remained constant. In the older sample, cortical gray matter volume decreased 